Cambridge University Press

0521537398 - Groups St Andrews 2001 in Oxford, Volume I

Edited by C. M. Campbell, E. F. Robertson and G. C. Smith

Excerpt

More information

\title{
PERMUTABILITY AND SUBNORMALITY IN FINITE GROUPS
}

\author{
MANUEL J. ALEJANDRE *, A. BALLESTER-BOLINCHES ${ }^{\dagger}$, R. ESTEBAN- \\ ROMERO ${ }^{\ddagger}$ and M. C. PEDRAZA-AGUILERA $\ddagger 1$ \\ * Centro de Investigación Operativa, Universidad Miguel Hernández, Avda. del Ferrocarril \\ s/n, 03202 Elche, Spain \\ † Departament d’Àlgebra, Universitat de València, C/ Doctor Moliner 50, 46100 Burjassot \\ (València), Spain \\ ¥ Departamento de Matemática Aplicada, E.U.I., Universidad Politécnica de Valencia, \\ Camino de Vera, s/n, 46071 Valencia, Spain
}

All groups considered in this report will be finite.

\section{Notation and terminology}

A group $G$ is said to be a $T$-group if every subnormal subgroup of $G$ is normal in $G$, that is, if normality is a transitive relation. These groups have been widely studied (see [10], [11], or [14]).

A subgroup $H$ of a group $G$ is said to be permutable (or quasinormal) in $G$ if $H K=K H$ for all subgroups $K$ of $G$. Permutability can be considered thus as a weak form of normality. The study of groups $G$ in which permutability is transitive, that is, $H$ permutable in $K$ and $K$ permutable in $G$ always imply that $H$ is permutable in $G$, has been a successful field of research in recent years. Such groups are called PT-groups. According to a theorem of Kegel [12, Satz 1], every permutable subgroup of $G$ is subnormal in $G$. Consequently, $P T$-groups are exactly those groups in which subnormality and permutability coincide; that is, those groups in which every subnormal subgroup permutes with every other subgroup. Therefore, every $T$-group is clearly a $P T$-group.

One could wonder what would happen if we did not require that every subnormal subgroup of a group $G$ permutes with any other subgroup of $G$, but only with a certain family of its subgroups. In this direction, those groups in which every subnormal subgroup of $G$ permutes with every Sylow $p$-subgroup of $G$ for each prime $p$ have sometimes been called $T^{*}$-groups (see [3]) or also $(\pi-q)$-groups (see [1]). Nevertheless, in recent years the expression PST-groups has become more popular for them. It was first used in [15]. Again, by a result of Kegel, each subgroup of a group $G$ permuting with every Sylow subgroup of $G$ is subnormal. Therefore, $P S T$-groups are exactly those groups in which permutability with Sylow subgroups is a transitive relation; that is, $G$ is a $P S T$-group when given $H \leq K \leq G$ such that $H$ permutes with every Sylow subgroup of $K$ and $K$ permutes with every Sylow subgroup of $G$, then $H$ permutes with every Sylow subgroup of $G$. It is clear that

$$
\{T \text {-groups }\} \subset\{P T \text {-groups }\} \subset\{P S T \text {-groups }\}
$$

\footnotetext{
${ }^{1}$ The work of the second and last authors is supported by Proyecto PB97-0674-C02-02 of DGICYT, MEC, Spain
} 
Cambridge University Press

0521537398 - Groups St Andrews 2001 in Oxford, Volume I

Edited by C. M. Campbell, E. F. Robertson and G. C. Smith

Excerpt

More information

and the above three classes are distinct.

The study of these classes of groups has undoubtedly constituted a fruitful topic of research in group theory, due to the efforts of many leading mathematicians. There are in essence three different ways of approaching the question of characterizing $T$-groups, $P T$-groups and $P S T$-groups. The aim of this survey is to provide a general perspective of these three lines. Note that every simple group belongs to these classes. Hence most of the papers on this topic frame their work in the soluble universe. In fact, as we shall see below, in some cases the solubility of the group appears in a spontaneous way.

\section{Characterizations based on the normal structure}

This is possibly the most classical way of studying these groups. The structure of soluble $T$-groups was determined by Gaschütz ([11]) in 1957. They are exactly the soluble groups $G$ with an abelian normal Hall subgroup $L$ of odd order such that $G / L$ is a Dedekind group and such that the elements of $G$ induce power automorphisms in $L$.

Some years later, in 1964, Zacher gave in [16] the corresponding theorem for soluble PT-groups: one just has to replace "Dedekind" by "nilpotent modular" in Gaschütz's theorem.

Finally, the structure of soluble PST-groups was obtained by Agrawal (see [1]), in a way similar to the Gaschütz and Zacher characterizations: $G$ is a soluble PSTgroup if and only if $G$ has an abelian normal Hall subgroup $L$ of odd order such that $G / L$ is nilpotent and the elements of $G$ induce power automorphisms in $L$.

As straightforward consequences of these theorems, one can state that the classes of soluble $T$-groups, soluble $P T$-groups and soluble $P S T$-groups are subgroupclosed. Recently, the second and third authors provided in [6, Theorem A] local versions of the above theorems.

\section{Characterizations based on the Sylow structure}

Several papers have explored the influence of the Sylow structure of a group on the condition of being a $P S T$-group, $P T$-group or $T$-group. The natural outcome of these investigations is the fact that in the soluble universe, the difference between these three classes is quite simply their Sylow structure. Therefore several unifying points of view for these classes have been obtained in the soluble universe.

These ideas were firstly used by Bryce and Cossey in [10]. With $p$ a prime, they defined the class $\mathcal{U}_{p}^{*}$ of $p$-supersoluble groups $G$ such that all the $p$-chief factors of $G$ form a single isomorphism class of $G$-modules. They proved that a soluble group $G$ is a $T$-group if and only if $G$ satisfies $\mathcal{U}_{p}^{*}$ for every prime $p$ and all its Sylow subgroups are $T$-groups. The first, second and fourth authors introduced in [2] the use of the class $\mathcal{U}_{p}^{*}$ for a single prime $p$ and the class $\cap_{p \in \mathbb{P}} \mathcal{U}_{p}^{*}$ to describe the classes of soluble $P T$-groups and $P S T$-groups. Many other relevant local definitions were introduced in [10] and in [2]. 
Cambridge University Press

0521537398 - Groups St Andrews 2001 in Oxford, Volume I

Edited by C. M. Campbell, E. F. Robertson and G. C. Smith

Excerpt

More information

This procedure of defining local versions in order to simplify the study of the global properties has shown itself to be very useful. The following three definitions are the main keys which allow us to describe the three classes we are working with in a natural way.

Definition 1 Let $G$ be a group and $p$ a prime. We say that $G$ :

1. Enjoys property $\mathcal{C}_{p}$ (see [14]) if each subgroup of a Sylow $p$-subgroup $P$ of $G$ is normal in the normalizer $N_{G}(P)$.

2. Satisfies property $\mathcal{X}_{p}$ (as in [7]) if each subgroup of a Sylow $p$-subgroup $P$ of $G$ is permutable in the normalizer $N_{G}(P)$.

3. Enjoys property $\mathcal{Y}_{p}$ (see [5]) if for all $p$-subgroups $H$ and $S$ of $G$ such that $H \leq S, H$ permutes with every Sylow subgroup of $N_{G}(S)$.

Note that the property $\mathcal{C}_{p}$ is inherited by subgroups. This fact follows from the abnormality of the Sylow normalizers in the group. By its definition, the class of $\mathcal{Y}_{p}$-groups is subgroup-closed as well. Nevertheless, proving that $\mathcal{X}_{p}$ is inherited by subgroups is of extreme difficulty. This fact was first observed by Beidleman, Brewster and Robinson in [7], but only as a consequence of a much stronger theorem.

The following theorem, obtained by the second and third authors in [5], summarizes the relationship between the three properties listed above and is crucial to having a global knowledge of their behaviour.

Theorem 1 [5, Theorem 3] A group $G$ satisfies $\mathcal{X}_{p}$ (respectively, $\mathcal{C}_{p}$ ) if and only if $G$ satisfies $\mathcal{Y}_{p}$ and its Sylow p-subgroups are modular (respectively, Dedekind).

This theorem is a consequence of the following $p$-nilpotence criterion, which was proved in the same paper:

Theorem 2 [5, Theorem 1] Let $p$ be a prime and let $G$ be a group with a modular Sylow p-subgroup $P$. Then $G$ is p-nilpotent if and only if $N_{G}(P)$ is p-nilpotent.

As a natural consequence of the above statements, the property $\mathcal{X}_{p}$ is inherited by subgroups. Not surprisingly, this fact, whose direct proof has been obtained only very recently, simplifies in a dramatic way the proofs of many of the existing theorems.

Robinson had proved in [14] that a group $G$ is a soluble $T$-group if and only if it satisfies property $\mathcal{C}_{p}$ for all primes $p$. Years later, Beidleman et al. proved in [7] that a group $G$ is a soluble $P T$-group if and only if it enjoys property $\mathcal{X}_{p}$ for every prime $p$. Finally, the second and third authors proved in [5] that a group $G$ is a soluble $P T$-group if and only if it satisfies $\mathcal{Y}_{p}$ for every prime $p$. One can see that in the theorem by Beidleman et al., a lot of effort is put into proving that every group satisfying $\mathcal{X}_{p}$ for every prime $p$ must be soluble. However, this assertion follows directly from the fact that $\mathcal{X}_{p}$ is closed under subgroups and factor groups.

Therefore, one can travel easily among the classes of soluble $P S T$-groups, soluble $P T$-groups and soluble $T$-groups (or among their local versions) just by changing the requirements on their Sylow $p$-subgroups. 
Cambridge University Press

0521537398 - Groups St Andrews 2001 in Oxford, Volume I

Edited by C. M. Campbell, E. F. Robertson and G. C. Smith

Excerpt

More information

Moreover, any hope of creating a similar landscape which could be valid also outside the soluble universe is soon dispelled. For instance, as soon as we have a local property $\mathcal{J}_{p}$ which is subgroup-closed and such that a finite group $G$ is a $P S T$-group if and only if $G$ satisfies $\mathcal{J}_{p}$ for every prime $p$, then it is possible to prove that $\cap_{p \in \mathbb{P}} \mathcal{J}_{p}$ is contained in the class of soluble groups. Hence we had better desist from our expectations.

Finally, the situation would be excellent if we could describe in a precise way the class of $\mathcal{Y}_{p}$-groups for a prime $p$, which is the central point which our description relies on. This wish was achieved in [5]:

Theorem 3 [5] A group $G$ is a $\mathcal{Y}_{p}$-group if and only if $G$ is either p-nilpotent or $G$ has abelian Sylow p-subgroups and $G$ satisfies $\mathcal{C}_{p}$.

\section{Characterizations based on embedding properties}

A strong connection between the classes described above and some embedding properties has recently been shown in several papers. Peng proved in [13] that a soluble group $G$ is a $T$-group if and only if every $p$-subgroup of $G$, for every prime $p$, is pronormal in $G$. In addition, Bianchi et al. presented in [9] the following embedding property: A subgroup $H$ of a group $G$ is said to be an $\mathcal{H}$-subgroup of $G$ if for all $g \in G, N_{G}(H) \cap H^{g} \leq H$. They proved that a group $G$ is a soluble $T$-group if and only if every subgroup of $G$ is an $\mathcal{H}$-subgroup of $G$. With a similar philosophy in mind, the second and third authors proved the following theorem.

Theorem 4 [4] Let $G$ be a group. The following statements are equivalent:

1. $G$ is a soluble T-group.

2. Every subgroup of $G$ is weakly normal in $G$.

3. Every subgroup of $G$ satisfies the subnormalizer condition.

A subgroup $H$ of $G$ is said to be weakly normal in $G$ if $H^{g} \leq N_{G}(H)$ implies that $g \in N_{G}(H)$ and is said to satisfy the subnormalizer condition if for every subgroup $K$ of $G$ such that $H$ is normal in $K$, it follows that $N_{G}(K) \leq N_{G}(H)$.

It is said that a subgroup $H$ of $G$ is hypercentrally embedded in $G$ if

$$
H / \operatorname{Core}_{G}(H) \leq Z_{\infty}\left(G / \operatorname{Core}_{G}(H)\right) .
$$

Beidleman and Heineken [8] proved that a soluble group $G$ is a PST-group if and only if every subnormal subgroup permutes with every Carter subgroup of $G$ and the subnormal subgroups of $G$ are hypercentrally embedded in $G$. The second and third authors showed in [6] that permutability with Carter subgroups could be removed, that is, a soluble group $G$ is a $P S T$-group if and only if every subnormal subgroup of $G$ is hypercentrally embedded in $G$. 
Cambridge University Press

0521537398 - Groups St Andrews 2001 in Oxford, Volume I

Edited by C. M. Campbell, E. F. Robertson and G. C. Smith

Excerpt

More information

PERMUTABILITY AND SUBNORMALITY IN FINITE GROUPS

\section{References}

[1] R.K. Agrawal "Finite groups whose subnormal subgroups permute with all Sylow subgroups" Proc. Amer. Math. Soc. 47 (1975), 77-83

[2] M. J. Alejandre, A. Ballester-Bolinches, M.C. Pedraza-Aguilera "Finite soluble groups with permutable subnormal subgroups" J. Algebra, 240 (2001), 705-721

[3] M. Asaad, P. Csörgö "On T*-groups" Acta Math. Hungar. 213 (1997), 235-243

[4] A. Ballester-Bolinches, R. Esteban-Romero "On finite T-groups" Preprint

[5] A. Ballester-Bolinches, R. Esteban-Romero "Sylow permutable subnormal subgroups of finite groups" Preprint

[6] A. Ballester-Bolinches, R. Esteban-Romero "Sylow permutable subnormal subgroups of finite groups II" To appear in Bull. Austral. Math. Soc.

[7] J.C. Beidleman, B. Brewster, D.J.S. Robinson "Criteria for permutability to be transitive in finite groups" J. Algebra, 222 (1999), 400-412

[8] J.C. Beidleman, H. Heineken "Finite soluble groups whose subnormal subgroups permute with certain classes of subgroups" Preprint

[9] M. Bianchi, A. Gillio Berta Mauri, M. Herzog, L. Verardi "On finite solvable groups in which normality is a transitive relation" To appear in J. of Group Theory.

[10] R.A. Bryce, J. Cossey "The Wielandt subgroup of a finite soluble group" J. London Math. Soc. 40 (1989), 244-256

[11] W. Gaschütz "Gruppen in deinen das Normalteilersein transitiv ist" J. reine angew. Math. 198 (1957), 87-92

[12] O.H. Kegel "Sylow-Gruppen und Subnormalteiler endlicher Gruppen" Math. Z. 78 (1962), 205-221

[13] T.A. Peng "Finite groups with pro-normal subgroups" Proc. Amer. Math. Soc. 20 (1969), 232-234

[14] D.J.S. Robinson "A note on finite groups in which normality is transitive" Proc. Amer. Math. Soc. 19 (1968), 933-937

[15] D.J.S. Robinson "The structure of finite groups in which permutability is a transitive relation", J. Aust. Math. Soc. 70 - 2 (2001), 143-149

[16] G. Zacher "I gruppi risolubili finiti in cui i sottogruppi di composizione coincidono con $i$ sottogruppi quasi-normali" Atti Accad. Naz. Lincei Rend. Cl. Sci. Fis. Mat. Natur. (8) 37 (1964), 150-154 
Cambridge University Press

0521537398 - Groups St Andrews 2001 in Oxford, Volume I

Edited by C. M. Campbell, E. F. Robertson and G. C. Smith

Excerpt

More information

\title{
(PRO)-FINITE AND (TOPOLOGICALLY) LOCALLY FINITE GROUPS WITH A CC-SUBGROUP
}

\author{
Z. ARAD* and W. HERFORT ${ }^{\dagger 1}$ \\ * Department of Mathematics, Bar-Ilan University, Ramat-Gan, Israel and \\ Department of Computer Science and Mathematics, \\ Netanya Academic College, Netanya, Israel \\ † Institut für Angewandte und Numerische Mathematik, \\ Technische Universität Vienna, Austria
}

\begin{abstract}
A proper subgroup $H$ of a group $G$ is called a CC-subgroup of $G$ if the centralizer $C_{G}(h)$ of $h \in H^{\#}=H \backslash\{1\}$ is contained in $H$. Such finite groups were partially classified by G. Frobenius, W. Feit , K. W. Gruenberg and O. H. Kegel, J.S. Williams, A.S. Kondrat'Iev, N. Iiyori and H. Yamaki, M. Suzuki, M. Herzog, Z. Arad, D. Chillag, Ch. Praeger and others.

In this report ${ }^{2}$, using the classification of finite simple groups, we give a complete list of all finite groups containing a CC-subgroup. As a corollary we classify infinite profinite groups, locally finite groups and certain classes of topological groups containing a CC-subgroup under certain conditions.
\end{abstract}

\section{Introduction}

Let $G$ denote a finite group. According to M. Herzog [18] a subgroup $M \leq G$ is a CC-subgroup ("centralizers contained"), if $C_{G}(m) \leq M$ for every $m \in M \backslash\{1\}$. The example with smallest cardinality is $G:=S_{3}$ with either $M:=\langle(123)\rangle$ or $M:=\langle(12)\rangle$ being a CC-subgroup. More generally, by the well known result of G. Frobenius, every Frobenius group has CC-subgroups either the kernel or any complement.

\subsection{Sketching the thread}

One finds the concept of a CC-subgroup (without calling it that) in work of W. FEIT describing doubly transitive groups which fix 3 letters (e.g. in [13]). He considered the situation of a group containing a CC-subgroup of order divisible by 3 and conjectured that $G$ is either Frobenius with kernel $M$ or $G=P S L\left(2,3^{n}\right)$ with $n \geq 1$, provided certain extra conditions hold.

In [25] M. Suzuki classified all groups containing a CC-subgroup of even order and in the proof of his Theorem 1 in [25] he said a group $G$ with a CC-subgroup to satisfying condition (c). M. SuZUKI found that such a group is either Frobenius,

\footnotetext{
${ }^{1}$ The second author would like to thank for greatful hospitality at the Bar-Ilan University, the Netanya Academic College and the Tel-Aviv University in February 2000

${ }^{2}$ The results of this report and their complete proofs are contained in [10]
} 
Cambridge University Press

0521537398 - Groups St Andrews 2001 in Oxford, Volume I

Edited by C. M. Campbell, E. F. Robertson and G. C. Smith

Excerpt

More information

$\operatorname{PSL}(2, q)$ for $q$ a power of 2 , or in today's terminology, $G$ is a Suzuki group over a field of characteristic 2. In [14] W. Feit and J. G. Thompson showed that if $M \cong C_{3}$ then $G$ is either an extension of a nilpotent group by either $A_{3}$ or $S_{3}$, or $G$ is an extension of a 2-group by $A_{5}$ or, third possibility, $G \cong P S L(2,7)$ and in [1] Z. ARAD generalized their result to classifying all groups containing a CC Sylow 3subgroup. M. HERzoG in [18] proved the aforementioned conjecture of Feit under additional assumptions. Z. ArAD in [2] and Z. Arad together with M. HerzoG in $[7,8]$, using results of W. B. STEWART [26], succeeded in determining a complete list of finite groups containing a CC-subgroup of order divisible by 3 .

Apparently, in an unpublished note of K. W. Gruenberg and O. H. Kegel and later by K. W. Gruenberg and K. W. RogGenkamp in [17] the notion of the prime graph of a finite group $G$ has been introduced to have vertices the primes dividing $|G|$ and edges $(p, q)$ whenever exist commuting elements $x, y \in G$ of respective orders $p \neq q$. Its connected components are denoted by sets of primes such that $2 \in \pi_{1}$ provided $G$ has even order. J.S. Williams 's Theorem 3 in [27] shows that to every odd component $\pi_{i}$ (only odd primes, $i>1$ ) there exists a $\pi_{i}$-Hall subgroup $M \leq G$, which is a an odd order CC-subgroup. The existence of such $M$ together with results in [17] show that the prime graph is disconnected if and only if the augmentation ideal decomposes as a $G$-module. The same authors introduce the notion of a 2-Frobenius group for a group $G$ containing a normal Frobenius group $H=K L, K \triangleleft G$ with $G / K$ again Frobenius group.

In Williams's Theorem 3 each such $M$ turns out to be nilpotent. Therefore it is desirable to give a complete description of groups containing a CC-subgroup including structural information on the group $M$ as well. Z. ARAD and D. ChillaG in $[4,5,6]$ continued classifying groups with a CC-subgroup. As a final result we present Theorem A below.

In [21] O. H. KegeL and B. A. F. Wehrfritz describe the situation of locally finite Frobenius groups and, answering a question originally posed by O. H. KEGEL, D. Gildenhuys, L. Ribes and W. Herfort provide a description of profinite Frobenius groups. Since in the profinite completion of the infinite dihedral group $C_{2} * C_{2}$ each of it's factors is a CC-subgroup and no normal complement to any of them exists, the extra condition of $M$ being a Hall-subgroup, has been included in the definition (see section 4.6 on profinite Frobenius groups in [24]). In [15] Yu. M. GORČAKOV gives a unifying result on algebraic and finite Frobenius groups. In [23] YU. N. MukHIN deals with certain topological Frobenius groups. We present results for profinite groups (Theorem B), locally finite groups (Theorem C) and topologically locally finite groups (every compact subset is contained in a compact subgroup - we denote this fact by $G \in[\mathrm{LF}]^{-}$) in Theorem D.

\section{Announcement of the results}

Following P.Hall [19] we say that $G$ satisfies $E_{\pi}$ if $G$ has a Hall $\pi$-subgroup denoted by $G_{\pi}$. $G$ satisfies $C_{\pi}$ if it satisfies $E_{\pi}$ and any two $S_{\pi}$-subgroups of $G$ are conjugate. $G$ satisfies $D_{\pi}$ if it satisfies $C_{\pi}$ and every $\pi$-subgroup of $G$ is contained in some $S_{\pi}$-subgroup of $G$. 
Cambridge University Press

0521537398 - Groups St Andrews 2001 in Oxford, Volume I

Edited by C. M. Campbell, E. F. Robertson and G. C. Smith

Excerpt

More information

The following result contains a complete classification of finite groups containing a CC-subgroup.

Theorem A Let $G$ be a finite group containing a $C C$-subgroup $M$. Let $\pi:=\pi(M)$. Then $G$ satisfies $D_{\pi}$. Furthermore we have one of the following four cases:

(1) $M$ is non-nilpotent and of even order and one of the following holds:

(a) $G$ is a Frobenius group with complement $M$;

(b) $G \cong P S L\left(2,2^{n}\right), n \geq 2$ and $M$ is solvable;

(c) $G \cong S z(q), q=2^{2 n+1}, n \geq 1$ and $M$ is solvable.

(2) $M$ is nilpotent of even order and one of the following holds:

(a) $G$ is a solvable Frobenius group with complement $M$;

(b) $G$ is a solvable Frobenius group with kernel $M$;

(c) $G \cong P S L\left(2,2^{n}\right), n \geq 2$ and $M$ is a 2-Sylow subgroup;

(d) $G \cong S z(q), q=2^{2 n+1}, n \geq 1$ and $M$ is a 2-Sylow subgroup.

(3) $M$ is non-nilpotent of odd order and one of the following holds

(a) $G$ is a solvable Frobenius group with complement $M$;

(b) $G \cong P S L(2, q), q \equiv 3 \quad(\bmod 4)$ and $M$ is solvable of odd order $|M|=$ $q \frac{q-1}{2}$

(4) $M$ is nilpotent of odd order and one of the following holds:

(a) $G$ is a Frobenius group with $M$ either kernel or complement;

(b) $G$ is simple non-abelian and $G$ and $M$ are classified in [27] and [28] ([29]) (see as well A.S. KONDRAT'IEV in [22]);

(c) $G$ is not simple. With $H:=(M)_{G}$ and $S:=H / F(H)$, one finds $S$ to be simple containing the CC-subgroup $M F(H) / F(H) \cong M$ ( $S$ and $M$ are classified in (4)(b)), and $F(H)$ and $G / H$ are $\pi_{1}$-groups.

(d) $G$ is a 2-Frobenius group, i.e., exists $F \triangleleft G$ such that $F M \triangleleft G$ is a Frobenius group with kernel $F$ and cyclic complement $M$, and exists cyclic $R \leq G$ with $M R$ Frobenius group (having kernel $M$ and cyclic complement $R$ ) and $G=F M R$.

Proof If $M \leq G$ is a CC-subgroup, then certainly the prime graph is disconnected. If $G$ is nilpotent then, using the classification of finite simple groups, the aforementioned result of Gruenberg and Kegel yields part of (b) and (d). If $M$ has even order then Suzuki's result yields (a) and the other part of (b). From now on $M$ is supposed to be of odd order and not nilpotent. Then $N_{G}(M)=M$ (else $N_{G}(M)$ is Frobenius and hence $M$ nilpotent) and [6] shows that $M=K L$ is a $\pi:=\pi(M)$ Hall subgroup, and at the same time is Frobenius with kernel $K$ and cyclic kernel 
Cambridge University Press

0521537398 - Groups St Andrews 2001 in Oxford, Volume I

Edited by C. M. Campbell, E. F. Robertson and G. C. Smith

Excerpt

More information

FINITE AND LOCALLY FINITE GROUPS WITH A CC-SUBGROUP

$L$. By the same paper $G$ satisfies $D_{\pi}$. From all this conclude that the prime graph of $G$ has at least 3 components and that $\pi$ contains at least two primes $p, q$ with $p$ dividing $q-1$. The list in (c) is found combining information on the order of $G$ from [12] and checking the tables in [27] and [28] for divisibility. Sporadic and alternating groups are excluded by using the fact that Hall subgroups involving precisely two primes $p, q$ (with $p<q \leq n$ if $G=A_{n}$ ) do not exist in neither $A_{n}$ [11] or any sporadic group [19].

Theorem B Let a profinite group $G$ contain a CC-Hall subgroup $M$. One of the following holds.

(i) $G$ is finite and contained in the list of Theorem A;

(ii) $G$ is infinite, $M$ is finite and one of the following holds:

(a) $G$ is a profinite Frobenius group with $M$ the Frobenius complement;

(b) With $H:=(M)_{G}$ the quotient $S:=H / F(H)$ is a finite simple group, $M F(H) / F(H) \cong M$ is a CC-subgroup of $S, F(H)$ is a nilpotent $\pi_{1}$ group, and $G / H$ is a finite $\pi_{1}$-group;

(c) $G$ is a profinite 2-Frobenius group, i.e., exists $F \triangleleft G$ open, such that $F M \triangleleft G$ is a profinite Frobenius group with kernel $F$ and finite cyclic complement $M$ and exists a finite $R \leq G$ with $M R$ a finite Frobenius group (kernel $M$, finite cyclic complement $R$ ) and $G=F M R$;

in (b) an isomorphic copy of $M$ is an odd order CC-subgroup of $S$; the group $S$ is as in Theorem A (4)(c);

(iii) $G$ and $M$ are both infinite and $G$ is a profinite Frobenius group with $M$ the Frobenius kernel.

Theorem C Let a locally finite group $G$ contain a CC-Hall subgroup $M$. One of the following holds.

(i) $G$ is finite and contained in the list of Theorem A;

(ii) $G$ is infinite, $M$ either contains an involution or is not locally nilpotent and one of the following holds:

(a) $G$ is a Frobenius group with $M$ either kernel or complement;

(b) $G \cong P S L(2, F)$, with $F$ a locally finite field;

(c) $G \cong S z(F), F$ a locally finite field of even characteristics and $M$ locally solvable;

(iii) $G$ is infinite, $M$ does not contain any involution and is locally nilpotent and one of the following holds:

(a) $G$ is a Frobenius group with $M$ either kernel or complement; 
Cambridge University Press

0521537398 - Groups St Andrews 2001 in Oxford, Volume I

Edited by C. M. Campbell, E. F. Robertson and G. C. Smith

Excerpt

More information

(b) $G$ is a locally finite 2-Frobenius group, i.e., exists $F \triangleleft G$ with $F M \triangleleft$ $G$ a locally finite Frobenius group (having kernel $F$ and locally cyclic complement $M$ ) and exists $R \leq G$ with $M R$ a locally finite Frobenius group (kernel $H$ and finite cyclic complement $R$ ) and $G=F M R$;

(c) For $H:=(M)_{G}$ the quotient $S:=H / F(H)$ is a locally finite simple group with $M F(H) / F(H)$ a CC-subgroup of $S, F(H)$ a nilpotent $\pi_{1}$ normal subgroup of $G$, and $G / H$ is a locally finite $\pi_{1}$ group. $S$ is a direct limit of groups each of them an extensions of a $\pi_{1}$-group by a simple group from (4)(b) in Theorem A.

With the help of Theorems B and $\mathrm{C}$ we establish a classification result for the class $[\mathrm{LF}]^{-}$of topologically locally finite groups (as introduced in [20]).

Theorem D Let $G \in[\mathrm{LF}]^{-}$be totally disconnected and neither be locally finite, nor compact. One of the following holds:

(i) $G$ is a topologically locally finite Frobenius group. $M$ is locally finite, isolated and complement and $F:=\left(G \backslash \bigcup_{g \in G} H^{g}\right) \cup\{1\}$ is a CC-normal subgroup of $G$ (the kernel);

(ii) $G$ is a topologically locally finite 2-Frobenius group, i.e., exists $F \triangleleft G$ open such that $F M \triangleleft G$ is topological Frobenius group ( $F$ the kernel and $M$ finite complement) and exists finite cyclic $R \leq G$ such that $M R$ is a finite Frobenius group with kernel $M$ and complement $R$ ( $G$ is topologically 2-Frobenius) and $G=F M R$;

(iii) $G$ is a topological Frobenius group with $M \triangleleft G$ an open $C C$-normal subgroup (the kernel) and it possesses a locally finite Frobenius-complement $H$, which is an isolated Hall subgroup of $G$;

(iv) $M$ is locally finite; for $H:=(M)_{G}$ the quotient $S:=H / F(H)$ is a locally finite simple group with $M F(H) / F(H) \cong M$ a CC-subgroup (as classified in Theorem $C), F(H)$ is a nilpotent normal $\pi_{1}$ subgroup of $G$, and $G / H$ is a locally finite $\pi_{1}$-group.

It should be desirable to extend our classification theorems to classes of groups containing algebraic groups as well as ours. The results of YU. M. GORČAKOV and Yu. N. MUKHIN indicate such a possibility.

\section{References}

[1] Z. ArAD , A classification of 3CC groups and applications to Glauberman-Scmidt theorem, J. Alg. 42, 176-180, (1976)

[2] Z. ARAD , A classification of groups with a centralizer condition, Bull. Austral. Math. Soc. 15, 81-85, (1976)

[3] Z. Arad \& D. Chillag, On Finite Groups Containing a CC-Subgroup, Arch. d. Math. 24, 225-234, (1977)

[4] Z. Arad \& D. Chillag, On Finite Groups Containing a CC-Subgroup, Arch. d. Math. 35, 401-405, (1980) 